\title{
PERCEPÇÃO DE UMA EQUIPE DE ENFERMAGEM SOBRE A UTILIZAÇÃO DO CHECKLIST CIRÚRGICO*
}

\author{
Perception of a nursing team about the use of surgical checklist \\ Percepción del equipo de enfermería sobre la utilización de lista de verificacion quirúrgica
}

Cátia Denise Perez Pereira Gomes ${ }^{1}$, Adriana Alves dos Santos ${ }^{2}$, Maria Elida Machado ${ }^{3}$, Patrícia Treviso ${ }^{4}$

RESUMO: Objetivo: Conhecer a percepção de profissionais de enfermagem que atuam em centro cirúrgico em relação à utilização do checklist cirúrgico. Método: Estudo exploratório, qualitativo. Os dados foram coletados de março a abril de 2015, por meio de entrevista gravada, com roteiro semiestruturado contendo dez perguntas, analisada sob a ótica da análise temática. Resultados: Participaram do estudo 13 profissionais de enfermagem. Os resultados foram organizados em três categorias: gerenciamento de risco em centro cirúrgico: dificuldades conceituais e na prática de trabalho; checklist de cirurgia segura e sua contribuição na prática de trabalho; e potencialidades e fragilidades na utilização do checklist de cirurgia segura. Conclusão: Profissionais de enfermagem percebem a necessidade de garantir a segurança do paciente, apontam que protocolos contribuem para a qualidade da assistência e dos serviços. O checklist é a principal ferramenta utilizada pela equipe visando à redução de danos e eventos adversos.

Palavras-chave: Gestão de riscos. Segurança. Lista de checagem. Enfermagem. Segurança do paciente.

ABSTRACT: Objective: To understand how nursing professionals who work in a surgical center, perceive the use of a surgical checklist. Method: Exploratory, qualitative study. The data were collected from March to April 2015, through recorded interviews with a semi-structured script containing ten questions. The data were analyzed by using thematic analyses. Results: The study included 13 nursing professionals. The results were organized into three categories: risk management in a surgical center: difficulties in concept and in work practice; checklist for safe surgery and its contribution to the working practice; strengths and weaknesses on using the checklist for safe surgery. Conclusion: Nursing professionals realize the need to ensure patient safety and think that protocols contribute to the quality of care and services. The checklist is the main tool used by the health team in order to reduce adverse events and damage.

Keywords: Risk management. Safety. Checklist. Nursing. Patient safety.

RESUMEN: Objetivo: Conocer la percepción de los profesionales de enfermería que trabajan en la sala de operaciones para el uso de la lista de verificación quirúrgica. Método: Estudio exploratorio, cualitativo. Se recogieron datos de marzo a abril de 2015 a través de entrevista grabada, semiestructurada que contiene diez preguntas. Los datos fueron analizados según el análisis temático. Resultados: Participaron del estudio 13 profesionales de enfermería. Los resultados fueron organizados en tres categorías: gestión de riesgos en el centro quirúrgico: dificultades conceptuales y prácticas del trabajo; lista de verificación para una cirugía segura y su contribución a la práctica del trabajo; potencialidades y debilidades de la lista de verificación de cirugía segura. Conclusión: Profesionales de enfermería reconocen la necesidad de garantizar la seguridad del paciente y señalan que los protocolos contribuyen a la calidad de la atención. La lista de verificación es la principal herramienta utilizada con el fin de reducir los eventos adversos y daños.

Palabras clave: Gestión de riesgos. Seguridad. Lista de verificación. Enfermería. Seguridad del paciente.

*Artigo resultante de Trabalho de Conclusão de Curso de graduação em Enfermagem do Centro Universitário Metodista IPA, finalizado em 2015. 'Graduanda em Enfermagem pela Centro Universitário Metodista IPA - Porto Alegre (RS), Brasil.

${ }^{2}$ Mestre em Biociências e Reabilitação pelo Centro Universitário Metodista IPA. Professora da Centro Universitário Metodista IPA - Porto Alegre (RS), Brasil.

${ }^{3}$ Doutora em Políticas Públicas e Formação Humana pela Universidade do Estado do Rio de Janeiro (UERJ). Professora do Centro Universitário Metodista IPA - Porto Alegre (RS), Brasil.

¿Doutora em Medicina e Ciências da Saúde pela Pontifícia Universidade Católica do Rio Grande do Sul (PUC-RS). Professora do Centro Universitário Metodista IPA - Porto Alegre (RS), Brasil. E-mail: ptreviso15@gmail.com Rua Doutor Tauphick Saadi, 33/601 - Bela Vista - CEP: 90470-040 - Porto Alegre (RS), Brasil.

Recebido: 14 dez. 2015 - Aprovado: 22 jul. 2016

DOI: $10.5327 / Z 1414-4425201600030004$ 


\section{INTRODUÇÃO}

A assistência cirúrgica tem sido um componente essencial do cuidado em saúde no Brasil e no mundo. À medida que as incidências de injúrias traumáticas, cânceres e doenças cardiovasculares aumentam, consequentemente há um impacto da intervenção cirúrgica nos sistemas de saúde pública. Para cada 25 pessoas, no mundo, 1 fará cirurgia, o que retrata a importância de se pensar a segurança na realização de um procedimento, pois metade das cirurgias realizadas apresenta complicações e morte como principais desfechos, sendo que $50 \%$ delas poderiam ser evitadas ${ }^{1}$.

Com o advento do gerenciamento de risco nas instituições de saúde, percebe-se melhoria na qualidade assistencial, pois as ações do gerenciamento de risco têm como foco a segurança dos pacientes, identificação das circunstâncias e oportunidades que colocam pacientes em risco, agindo na sua prevenção e controle ${ }^{2}$. Os programas de qualidade em serviços de saúde estimulam ações de observação dos padrões de conformidade, na perspectiva de melhoria do desempenho da organização e da segurança dos pacientes internados ${ }^{3}$.

Diante deste contexto, acredita-se que o gerenciamento de risco possibilita aos profissionais de enfermagem avaliar o cuidado oferecido ao paciente, observando e propondo melhores práticas que minimizem problemas ou até mesmo antecipando-os-.

Em outubro de 2004, a Organização Mundial de Saúde (OMS) lançou a Aliança Mundial para a Segurança do Paciente, que teve como objetivo despertar a consciência profissional e o comprometimento político para uma melhor segurança na assistência em saúde e para o desenvolvimento de políticas públicas que induzam a boas práticas assistenciais. Dentre os desafios globais que fomentam o comprometimento mundial da cultura de segurança do paciente está a preocupação com os procedimentos clínicos e cirúrgicos seguros, que dispõem de fundamentos essenciais para assistência em saúde, aumentando o padrão de qualidade, evitando eventos adversos e danos ao paciente ${ }^{1}$.

O evento adverso ocorre quando há falha de processos na organização dos serviços, bem como a falta de liderança ou de condutas que modifiquem uma realidade que pode causar danos permanentes e até mesmo a morte 4 . Dessa forma, quando há um evento adverso, toda a estrutura organizacional sofre consequências sejam elas sociais, econômicas ou materiais 5 .

No Brasil, o Ministério da Saúde, em parceria com a Organização Pan-americana e a Organização Mundial de Saúde, foi responsável pela apresentação do Manual Cirurgias
Seguras Salvam Vidas, que visa colaborar com a prática preventiva de riscos aliada à qualidade de assistência aos pacientes ${ }^{6}$.

Esse manual apresenta uma lista de verificação de segurança cirúrgica (checklist), desenvolvido por especialistas e dividido em três etapas, descritas como: identificação (antes da indução anestésica), confirmação (antes da incisão cirúrgica) e registro (antes do paciente sair da sala de operações). Na prática assistencial, nem sempre essa ferramenta tem contribuído para uma melhor comunicação, no entanto, proporciona mais segurança ao procedimento ${ }^{6,7}$. Além disso, os profissionais estão mais sensíveis às questões da segurança do paciente e manifestam opinião favorável quanto à aplicação do checklist cirúrgico, bem como a sua implementação nos serviços de saúde ${ }^{8}$.

A OMS sugere que o checklist deva ser realizado pelo enfermeiro, porém, pode ser realizado por outro profissional de saúde devidamente habilitado para esse fim e envolvido com o procedimento cirúrgico proposto?.

Em estudo multicêntrico realizado com oito instituições, no qual o checklist cirúrgico foi adotado em caráter experimental, foi evidenciado que o uso desse instrumento quase dobrou as chances de os pacientes receberem o tratamento adequado, isso é, livre de danos. Além disso, a aplicação do checklist reduziu em $47 \%$ a mortalidade no pós-operatório e em $11 \%$ as complicações cirúrgicas ${ }^{10}$.

$\mathrm{O}$ entendimento dos motivos pelos quais os incidentes ocorrem facilita a elaboração de estratégias e ações para a redução dos riscos, aumentando a segurança do paciente. A resposta organizacional frente ao incidente ocorrido proporciona o aprendizado, gerando mudanças no sistema de melhoria de qualidade em saúde. A qualidade nos serviços de saúde é um elemento determinante, que assegura e controla os riscos aos quais os pacientes estão submetidos ${ }^{11}$. As instituições de saúde percebem suas fragilidades, a fim de identificar a fonte de eventuais erros e corrigi-los ${ }^{12}$.

A partir das considerações sobre o uso do checklist de cirurgia segura, este estudo propôs responder a seguinte pergunta de pesquisa: qual a percepção dos profissionais de enfermagem que atuam em centro cirúrgico acerca do checklist de cirurgia segura?

O presente estudo teve como objetivo geral conhecer a percepção de profissionais de enfermagem que atuam em centro cirúrgico em relação à utilização do checklist; e como objetivos específicos, conhecer a percepção dos profissionais de enfermagem quanto ao gerenciamento de risco na prática de trabatho, conhecer as potencialidades e fragilidades na utilização do checklist de cirurgia segura e identificar os benefícios da utilização do checklist de cirurgia segura para a equipe de enfermagem. 


\section{MÉTODO}

Trata-se de uma pesquisa exploratória com abordagem qualitativa. Os dados foram coletados em um centro cirúrgico de um hospital privado de médio porte, que realiza cerca de 150 cirurgias ao mês, no município de Porto Alegre, Rio Grande do Sul.

Os critérios de inclusão dos sujeitos foram: profissionais de enfermagem que atuam exclusivamente no centro cirúrgico, nos turnos da manhã e tarde, e que consentiram em participar do estudo por meio da leitura e assinatura do Termo de Consentimento Livre e Esclarecido (TCLE). Foram excluídos os profissionais de enfermagem que possuíam experiência inferior a três meses, afastamento do trabalho por problemas de saúde, férias ou folgas por ocasião da coleta de dados. Os profissionais que trabalhavam no turno da noite não são exclusivos do setor, trabalhando por regime de escala e, portanto, não atendiam aos critérios de inclusão.

Para garantir o anonimato dos sujeitos da pesquisa, os participantes foram identificados por letras, seguidas de números arábicos, como por exemplo, E1 e T1 - sendo que $\mathrm{E}$ refere-se aos enfermeiros e $\mathrm{T}$ aos técnicos de enfermagem - , seguidos dos números correspondentes à ordem das entrevistas. A coleta de dados ocorreu nos meses de março e abril de 2015 , por meio de um roteiro semiestruturado contendo nove perguntas, sendo três fechadas que tratavam do perfil dos entrevistados (categoria profissional, turno de trabalho e tempo de atuação) e sete abertas, sobre gerenciamento de risco e checklist no que se refere aos benefícios, dificuldades, facilidades na utilização e da comunicação entre os profissionais. Acredita-se que essas questões foram suficientes para atender aos objetivos da pesquisa. Os dados foram coletados por meio de gravação em MP3 e posteriormente transcritas.

O tratamento dos dados se deu a partir da análise temática $^{13}$. Primeiramente, as respostas foram agrupadas por temas, a seguir foram identificadas as seguintes categorias de análise: gerenciamento de risco em centro cirúrgico: dificuldades conceituais e na prática de trabalho; checklist de cirurgia segura e sua contribuição na prática de trabalho; e potencialidades e fragilidades do checklist de cirurgia segura.

O projeto de pesquisa foi submetido e aprovado pelos comitês de ética e pesquisa das instituições envolvidas, proponente e coparticipante, sob os números de pareceres 924.294 e 947.103, respectivamente, seguindo a Resolução n ${ }^{\circ}$ 466/ 12 do Conselho Nacional de Saúde ${ }^{14}$.

\section{RESULTADOS E DISCUSSÃO}

Participaram da pesquisa 13 profissionais de enfermagem, sendo 4 enfermeiros e 9 técnicos de enfermagem.

Em relação ao tempo de experiência em centro cirúrgico, dois enfermeiros possuem entre um e cinco anos de experiência, um enfermeiro entre cinco e dez anos e um acima de dez anos. Já entre os técnicos de enfermagem, dois dos profissionais possuem entre um e cinco anos de experiência, cinco deles possuem entre cinco e dez anos e dois técnicos possuem acima de dez anos de experiência.

Os resultados estão apresentados segundo as categorias de análise.

\section{Gerenciamento de risco em centro cirúrgico: dificuldades conceituais e na prática de trabalho}

De modo geral, os profissionais de enfermagem em centro cirúrgico possuem dificuldades em definir gerenciamento de risco, entretanto, percebem sua importância como algo relacionado diretamente com a segurança tanto do paciente como do trabalhador, estando vinculado às normas e aos protocolos institucionais. Ao conceituar gerenciamento de risco, os profissionais o descrevem como um conjunto de ações que antecipam problemas futuros, dessa forma reduzindo e prevenindo danos, como podemos observar nas falas a seguir.

[...] Gerenciamento de risco são todos os protocolos e todas as atitudes para o cuidado do paciente, para evitar o risco, todas as atuações que nós temos dentro do bloco são para evitar o risco do paciente [...] (E3)

Essa fala ilustra adequadamente a concepção de que o gerenciamento de risco promove de maneira sistematizada o cuidado do paciente, identificando possíveis eventos que possam prejudicá-lo, fortalecendo a segurança e a qualidade dos processos $^{15}$.

É possível perceber também nas respostas dos entrevistados algumas das recomendações da OMS, relacionadas à segurança do paciente, como identificação correta, risco para quedas, administração de medicamentos, localização e lateralidade corretos, entre outros. Acredita-se que questões relacionadas à segurança perpassam por diversas fases 
do cuidado, desde a internação até a alta do paciente independente do local onde ele está inserido. A fala a seguir ilustra esta reflexão:

[...] São os cuidados que devemos ter com o paciente, não deixá-lo cair, não fazer medicação errada, ter o cuidado direto com o cliente, é receber bem o paciente e proporcionar conforto a ele e aos familiares $[\ldots](\mathrm{T} 3)$

Mesmo com dificuldades em conceituar gerenciamento de risco em si, os profissionais tem o entendimento sobre processos que envolvem a segurança do paciente e acreditam que gerenciar risco perpassa por ações que antecedem e previnem a ocorrências de erros relacionados à saúde.

[...] Gerenciamento de risco é uma forma que a instituição tem de prever acontecimentos, alguma coisa que vá acarretar problemas futuros ou pro paciente ou pro familiar e equipe. Eu entendo que é uma prevenção de alguma coisa mais grave $[\ldots](\mathrm{T} 9)$.

Essa percepção sobre o caráter sistêmico do gerenciamento de risco é sustentada à luz da literatura, a qual aponta que a segurança do paciente está diretamente relacionada com a redução e/ou atenuação de atos considerados inseguros que estão atrelados ao sistema de saúde, visando ao emprego das melhores práticas com propósito de obter os resultados esperados ${ }^{12}$.

\section{Checklist de cirurgia segura: contribuição na prática de trabalho}

O checklist cirúrgico é uma ferramenta útil para diminuir eventos adversos em hospitais, porém, sua implantação efetiva é desafiadora ${ }^{16}$. Trata-se de um instrumento para a segurança do paciente, podendo ser utilizado em situações completamente distintas, antecipando eventuais danos e promovendo a qualidade assistencial, como evidenciamos na fala a seguir:

[...] O checklist, é a certeza de que estamos fazendo a coisa certa, com o paciente certo, na hora certa. E a confirmação de que tudo tá certo [...] o checklist dá certeza de que a cirurgia é segura, o paciente sabe que tá no lugar certo, sendo operado pelo médico certo, pela equipe certa [...] (T5)
A literatura sustenta que essa ferramenta tem como objetivo reduzir consideravelmente os riscos mais comuns e evitáveis durante o perioperatório, e se correlaciona com o período de tempo relativo ao fluxo normal da cirurgia a partir de uma sequência de ações estabelecidas ${ }^{17}$.

Após análise reflexiva das falas, percebe-se que o checklist, além de garantir a segurança cirúrgica do paciente, também qualifica o trabalho da equipe envolvida no processo operatório, promovendo, por sua vez, o diálogo entre seus atores e a interface entre os profissionais de enfermagem e a equipe multiprofissional, conforme se observa na fala a seguir:

[...] conversamos mais sobre as dificuldades do paciente, as observações que a gente tem para com ele, se tem alergia, antes não era falado sobre isso, se o médico quer antibiótico [...] antes do checklist não havia esse questionamento, essas perguntas, esse intercâmbio entre a equipe [...] (T3)

A utilização do checklist cirúrgico como parte do processo de trabalho ratifica a centralidade do cuidado no paciente, sendo uma ferramenta com potencial para a qualificação da assistência de enfermagem ${ }^{7}$. Acredita-se que a implementação de protocolos, como o checklist, nos serviços promova a comunicação efetiva entre os profissionais da equipe multiprofissional.

No entanto, ao mesmo tempo em que tal ferramenta é elemento de segurança, pode também a sua utilização gerar constrangimentos para a equipe, o que chama a atenção nas falas de T1 e T6:

[...] médicos antigos, eles não levam muito a sério o checklist $[\ldots]$ isso acaba deixando a gente um pouco mais encabulado, não consegue fazer o trabalho direito $[\ldots](\mathrm{T} 1)$

[...] não funciona muito bem às vezes, é difícil fazer com que funcione tudo, nem sempre é bem aceito $[\ldots](\mathrm{T} 6)$

Apesar de ser um desafio enfrentado pela equipe de enfermagem, esse aspecto deve ser levado em conta, pois ao realizar o checklist, todos os integrantes da equipe participam ativamente da comunicação durante a confirmação dos itens, comunicando suas ações e preocupações a todos na sala cirúrgica ${ }^{7}$. 


\section{Potencialidades e fragilidades na utilização do checklist de cirurgia segura}

Na percepção dos profissionais de enfermagem estudados, o principal ganho em relação ao checklist está na utilização dessa ferramenta para tomada de decisão e atitudes que visam à segurança do paciente e equipe, evitando erros, proporcionando ações efetivas, como percebe-se nas falas a seguir:

[...] Contribui para procedimento correto, local, equipe certa, se não houve erro no tratamento[...](E1)

[...] A facilidade é que evita mesmo o erro, de chamar paciente errado, fazer cirurgia errada, isso facilitou muito para não acontecer esses erros [...] (T8)

Se realizada de maneira completa e fidedigna pelas equipes, a lista de verificação proporciona a observação das etapas críticas dos procedimentos cirúrgicos, reduzindo as complicações e a mortalidade, prevenindo as infecções de sítio cirúrgico, erros relacionados à cirurgia, melhorando a eficiência das equipes e anestesiologia ${ }^{18}$.

Cabe ainda citar como uma das potencialidades voltadas para o uso do checklist o papel do enfermeiro como gestor no processo de cuidado, viabilizando um melhor trabalho em equipe, como podemos perceber na fala a seguir:

[...] os enfermeiros abraçaram a causa, isso foi um ponto positivo, porque mostra como faz as vias de gerente, com toda a equipe multiprofissional. Em todas as situações o enfermeiro é esse facilitador, aqui a realidade foi bem positiva da figura do enfermeiro [...] (E4)

A gestão é fundamental no trabalho do enfermeiro, por atuar juntamente com diversas equipes multiprofissionais. O enfermeiro não deve supervalorizar o controle, hierarquia e a impessoalidade, mas participar de um processo de trabalho baseado no diálogo, na participação e no debate junto à equipe para melhores tomadas de decisões ${ }^{19}$.

Dentre as dificuldades apontadas pelos técnicos e enfermeiros acerca do checklist destaca-se a resistência e banalização da equipe médica em relação a sua execução, como podemos analisar nas falas a seguir:

[...] As dificuldades são com relação aos médicos, os médicos são mais resistentes ao responder o checklist $[\ldots](\mathrm{T} 2)$
[...] o médico que às vezes não aceita bem dá risada $[\ldots]$ às vezes o médico naquela angústia de querer fazer de uma vez a cirurgia não tem paciência pro checklist $[\ldots]$ (T4)

A maior dificuldade encontrada para o bom desempenho da equipe cirúrgica está na própria equipe. Cirurgiões, anestesistas, enfermeiros e outros profissionais envolvidos com o procedimento cirúrgico devem manter um bom relacionamento e comunicação efetiva. A equipe que trabalha unida com o propósito de aplicar seus conhecimentos e habilidades em benefício do paciente acaba por prevenir consideravelmente as complicações relacionadas ao ato cirúrgico que possam ameaçar a vida ${ }^{20}$.

Os resultados desta pesquisa ratificam os achados de outros estudos semelhantes quanto às fragilidades e pontencialidades do checklist de cirurgia segura. No que tange às fragilidades, há um grande caminho a ser percorrido permeando a gestão dos processos de trabalho no centro cirúrgico, bem como no papel do enfermeiro junto à equipe multiprofissional.

\section{CONSIDERAÇÕES FINAIS}

Os profissionais de enfermagem em centro cirúrgico percebem a necessidade de garantir a segurança do paciente. A percepção dos profissionais de enfermagem sobre o gerenciamento de risco na prática de trabalho em centro cirúrgico está diretamente relacionada às melhores práticas assistenciais, instituição de protocolos que propiciem prevenção de eventos adversos e qualificação assistencial, a partir da redução dos erros e, consequentemente, dano ao paciente. $O$ entrosamento entre a equipe, a redução da chance de erros e a participação ativa dos enfermeiros aparecem como as principais potencialidades do checklist. Em contrapartida, a resistência por parte da equipe médica é sinalizada como uma das principais fragilidades. O checklist contribui não apenas para a segurança do paciente, mas para a segurança da própria equipe à medida que essa ferramenta permite identificar o paciente, o procedimento, a lateralidade, entre tantos outros aspectos. Processos educativos são necessários para sensibilizar a equipe quanto à importância desse instrumento de verificação, bem como promover maior integração da equipe multiprofissional, reforçando a atuação conjunta na prestação de uma assistência segura e de qualidade ao paciente cirúrgico. 


\section{REFERÊNCIAS}

1. Organização Mundial da Saúde. Aliança Mundial para a Segurança do Paciente. Segundo desafio global para a segurança do paciente: cirurgias seguras salvam vidas. Rio de Janeiro: Organização -Mundial daSaúde; 2009.

2. Silva LFN. Reorientação do gerenciamento de risco hospitalar do Instituto Nacional de Traumatologia e Ortopedia (dissertação). Rio de Janeiro: FIOCRUZ; 2009

3. Lima HO, Dutra ECD. 0 gerenciamento de riscos na saúde: aplicação na atenção hospitalar. Rev Adm Hosp Inovação Saúde; 2010:87-90 [acesso em 24 jan 2015]. Disponível em: http://revistas.face.ufmg. br/index.php/rahis/article/view/1114

4. Branco -Filho JRC. Construindo um modelo de segurança do paciente. Prát Hosp. 2010;13(74):8-9.

5. Universidade de São Paulo. Faculdade de Medicina. Hospital das Clínicas da Faculdade de Medicina de Ribeirão Preto. Cartilha de gerenciamento de riscos e segurança do paciente. Ribeirão Preto: USP; 2010 [acesso em 05 jan 2015]. Disponível em: http://www. sbrafh.org.br/site/public/temp/510f0a460507f.pdf

6. Monteiro F, Silva LR. “Checklist” lista de verificação de segurança cirúrgica: avaliação e intervenção. Rev Ciênc Méd Biol. 2013;12(Esp):482-5.

7. Pancieri AP, Santos BP, Avila MAG, Braga EM. Checklist de cirurgia segura: análise da segurança e comunicação das equipes de um hospital escola. Rev Gaúcha Enferm. 2013;34(1):71-8.

8. Valido SCN. Checklist cirúrgica: contributo para uma intervenção na área da segurança do doente (dissertação). Évora: Universidade de Évora; 2011 [acesso em 21 jan 2015]. Disponível em: http://hdl. handle.net/10400.21/1594

9. Gomes JRAA, Melanda VS. Elaboração de rotinas para uma enfermagem de excelência em centro cirúrgico. Rev SOBECC. 2012;17(2):48-55.

10. Schalack WS, Boermeester MA. Patient safety during anaesthesia: incorporation of the WHO safe surgery guidelines into clinical practice. Curr Opin Anaesthesiol. 2010;23(6):754-8.

11. Belo AC. A criança segura em serviço de saúde. In: Ministério da Saúde. Assistência segura: uma reflexão teóricaaplicada prática. Brasilia: Anvisa;2013.
12. Grigoleto ARL, Gimenes FRE, Avelar MCQ. Segurança do cliente e as ações frente ao procedimento cirúrgico. Rev Eletr Enferm. 2011;13(2):347-54

13. Gomes R. Análise e interpretação de dados de pesquisa qualitativa. In: Deslandes SF, Gomes R, Minayo MCS (Orgs.). Pesquisa social: teoria, método e criatividade. 26 ed. Petrópolis: Vozes; 2007. p. 79-108.

14. Brasil. Resolução no 466, de 12 de dezembro de 2012. Diretrizes e normas regulamentadoras de pesquisas envolvendo seres humanos. Brasília: Diário Oficial da União; 2013. Seção 1. p. 59-62.

15. Lima CA, Faria JS, Machado APN, Gonçalves RPF, Teixeira MG, Oliveira RS, et al. Gestão de risco hospitalar: um enfoque na qualidade e segurança do paciente. Rev Eletr Gestão Saúde. 2014;2682-76 [acesso em 24 jan 2015]-. Disponível em: http://gestaoesaude.unb.br/index.php/gestaoesaude/article/ viewFile/692/pdf

16. Freitas MR, Antunes AG, Lopes BNA, Fernandes FC, Monte LC, Gama ZAS. Avaliação da adesão ao checklist de cirurgia segura da OMS em cirurgias urológicas e ginecológicas, em dois hospitais de ensino de Natal, Rio Grande do Norte, Brasil. Cad Saúde Pública. 2014;30(1):137-48.

17. Porto KLH. A segurança do paciente na utilização do checklist. Enferm Rev. 2014;17(2)103-15.

18. Araújo MPS, Oliveira AC. Contribuições do programa "cirurgias seguras salvam vidas" na assistência ao paciente cirúrgico: revisão integrativa. Rev Enferm UFPE. 2015;9(4):7448-57.

19. Gomes LC, Dutra KE, Pereira ALS. 0 enfermeiro no gerenciamento do centro cirúrgico. Rev Eletr Fac Metodista Granbery. 2014;16:1-21 [acesso em 25 mar 2015]-. Disponivel em: http://re.granbery.edu. br/artigos/NTEy.pdf

20. Panesar SS, Noble DJ, Mirza SB, Patel B, Mann B, Emerton M, et al. Can the surgical checklist reduce the risk of wrong site surgery in orthopaedics? Can the checklist help? Supporting evidence from analysis of a national patient incident reporting system. J Orthop Surg Res. $2011 ; 6: 18$. 